\title{
Lipoprotein particle sizes in patients undergoing kidney transplantation
}

\author{
Breza J Jr' ${ }^{1}$, Durackova $Z^{2}$, Payer J ${ }^{3}$, Dukat $A^{3}$, Breza J Sr${ }^{1}$ \\ Department of Urology, Comenius University, Bratislava, Slovakia. janbreza@gmail.com
}

\begin{abstract}
Asymptomatic atherosclerotic disease is prevalent in the middle-aged group of kidney transplant recipients. In order to improve the estimation of their cardiovascular risk, dyslipidaemia pattern is very important to be evaluated. Knowledge of the lipid particle spectrum in patients undergoing kidney transplantation could help the clinicians to timely start intervention and prevention of atherosclerosis with an early hypolipidemic statin treatment (Tab. 2, Ref. 20). Text in PDF www.elis.sk.

KEY WORDS: kidney transplantation, dyslipidaemia, atherosclerosis.
\end{abstract}

\section{Introduction}

Middle-aged patients exhibit a high prevalence of asymptomatic atherosclerosis $(1,2)$. This is the case also for the group of patients waiting for kidney transplantation because of their chronic kidney disease (3). Patients after kidney transplantation often suffer from dyslipidaemia due to the side-effects of medication, weight gain or diet $(4,5)$. There is large amount of data about lipid changes after renal transplantation, but not at the time of the procedure (6). In order to know the lipoprotein particle sizes in the serum of these patients prior the procedure, we performed a pilot study evaluating the complete lipid spectrum and measuring the sizes of lipid particles.

\section{Patients and methods}

In four patients, two men and two women with the end-stage kidney disease (non-smokers, no alcohol addicts), lipoprotein sizes were evaluated with Lipoprint Quantimetric System (7) before kidney transplant surgery. All of them were untreated with hypolipidemic therapy. Results were compared to the matching group of healthy subjects. Characteristics of the studied sample are shown in Table 1.

\section{Statistical analysis}

The data were provided as the means \pm standard deviation. The differences between quantitative variables were compared using the t-test for variables with normal distribution. Differences with p values less than 0.05 were considered as statistically significant. For the statistical analysis, the GraphPad Prism version 5.03 for Windows was used.

${ }^{1}$ Department of Urology, Comenius University, Bratislava, Slovakia, ${ }^{2}$ Institute of Chemistry, Biochemistry and Clinical Biochemistry, Comenius University, Bratislava, Slovakia, and ${ }^{3} 5$ th Department of Internal Medicine, Comenius University, Bratislava, Slovakia
Ethics

The study was approved by the ethical committee, all patients signed the informed consent, and complied with the ethical investigation principles outlined in the Declaration of Helsinki.

\section{Results}

Before kidney transplantation, patients with end-stage kidney disease had a significantly different lipoprotein spectrum than the control subjects. They did not differ in the low-density cholesterol (LDL-C) particle sizes, but had a significantly lower high-density cholesterol (HDL-C). They revealed also another lipid pattern with a significantly higher spectrum of very low -density lipoproteins (VLDL) and large and middle size intermediate (IDL) lipoprotein levels. Small IDL particles did not differ from the controls of the same age. The obtained results are shown in Table 2. There were no significant correlations of lipid parameters with other measured parameters of kidney function.

Thus, the measurement of the whole lipid spectrum even before kidney transplantation may be a part of useful laboratory evaluations for the future prognosis.

\section{Discussion}

Epidemiologic studies clearly showed that dyslipidaemia represents one of the major risk factors for atherosclerosis and its complications for atherothrombosis $(8,9)$. Unsatisfactory preventive measures contribute to a very high residual cardiovascular risk (10).

Pathogenesis of the post-transplant atherosclerotic cardiovascular disease most likely involves common risk factors (11). Although dyslipidaemia and cardiovascular diseases are common complications of kidney transplantation, the casual association of dyslipidaemias with cardiovascular risk has not been proven in these groups of patients (12). 
Tab. 1. Characteristics of the sample.

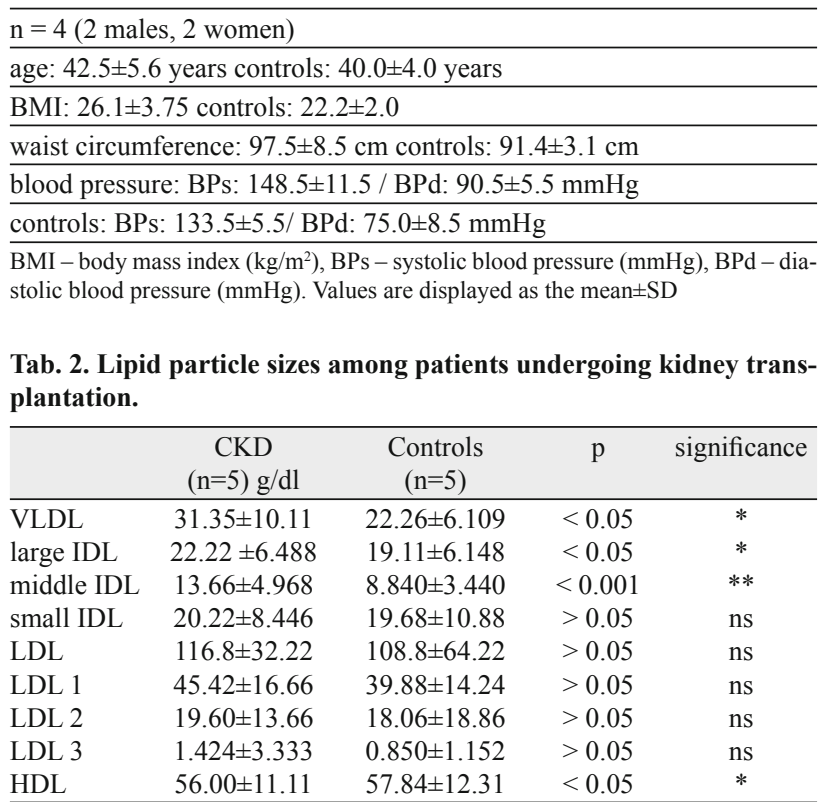

CKD - chronic kidney disease, VLDL - very low-density lipoproteins, IDL - intermediate density lipoproteins, LDL - low density lipoproteins, HDL - high density lipoproteins, ns - non-significant statistical significance: $* \mathrm{p}<0.05, * * \mathrm{p}<0.001$

Kidney transplantation is the treatment of choice for most patients with the end-stage kidney disease. Successful transplantation improves their quality of life and reduces their mortality. Patients with end-stage kidney disease suffer from significant comorbidities (12). Cardiovascular disease is the leading cause of morbidity and mortality in the patients after renal transplantation (13). Death from a cardiovascular disease is also the most common cause of the loss of the draft (14). Potential kidney transplant recipients have to be evaluated in order to decrease their subsequent perioperative and postoperative risks and improve their later prognosis (15).

The high rate of cardiovascular deaths in this group of patients is partly due to the large proportion of diabetic comorbidity in the population with the end-stage kidney disease and markedly increased cardiovascular risk (16).

An interesting finding was the detected increased presence of VLDL and large and middle size IDL particles. They are also carrying their certain atherogenic risk potential (17). Together with the low levels of protective HDL-cholesterol levels, with their dysfunctional capabilities, they participate in the increased global atherogenic risk.

Metabolism of lipoproteins might be disturbed by uraemia. After kidney transplantation, the metabolism of lipoproteins is affected also by immunosuppression, hypotensives and other drugs, which may further deepen the changes described.

For the laboratory evidence of lipoprotein metabolism disorders, a clinical equivalent may be found. From the clinical point of view, ischemia in generalized atherosclerosis, which is a significant cardiovascular risk in recipients of transplanted kidneys, can also adversely affect other organ systems in the recipient and the transplanted kidney itself. Ischemia resulting from atheroscle- rotic changes in the blood vessels after kidney transplantation may, in combination with a rejection response to the transplanted kidney, lead to a serious disorder of the function of the transplanted kidney with a possible loss of this organ. These effects could be pronounced even more after a heart transplantation. Another example of the negative influence of ischemia caused by generalized atherosclerosis are functional and organic changes of the organs of the small pelvis. Reconstruction of the lower urinary tract and its adequate function is a condition and a part of successful kidney transplantation.

Normal function of the lower urinary tract contributes significantly to the success of the kidney transplantation. On the other hand, dysfunction of the lower urinary tract, caused by ischemia can lead to the pain or even loss of the transplanted kidney.

\section{Conclusions}

Patients awaiting kidney transplantation bear a higher risk of premature atherosclerosis than the control patients also due to dyslipidaemia (18). Its characteristics differ from the dyslipidemic profile seen in the patients after kidney transplantation.

There was a small increase of atherogenic LDL-cholesterol, however, other atherogenic lipid particles such as VLDL and IDL lipids were present in significantly higher concentrations. As they also carry a certain atherogenic potential, hypolipidemic treatment predominantly with statin is indicated even before transplantation (19). Concomitant constant findings showed also lower HDLcholesterol levels indicating the usefulness of an early hypolipidemic treatment (20).

All of the aspects and consequences of hyperlipidaemia mentioned above should be the incentive for a complete laboratory and clinical examination focused on the possible disorders of lipoprotein metabolism in patients waiting for kidney transplantation as well as in those after transplantation. The proof of changes in lipoprotein metabolism should be an indication for beginning a prophylactic treatment with hypolipidemics.

These preliminary data need to be confirmed in a larger number of patients, however, it seems to be important to include also laboratory measurements of the lipid particle sizes before kidney transplantation. This evaluation is efficient and cost effective. Early starting of hypolipidemic treatment strategies is mandatory in order to decrease their high atherogenic risk, possibly not in the statin monotherapy, but with combinations of hypolipidemics. The prevalence and ability to modify dyslipidaemias is an important intervention for improving the outcomes after kidney transplantation.

Limitations of the study: This is a pilot study with several monitored patients. These preliminary results have to be proven in the larger patient groups.

\section{References}

1. Andersson Ch, Lyass A, Vasan R et al. Long-term risk of cardiovascular events across a spectrum of adverse major plasma lipid combinations in the Framingham Heart Study. Am Heart J 2014; 168: 878-883.

2. Yusuf S (Ed). Evidence based cardiology. BMJ. Book London; 2003: 968. 


\section{$130-132$}

3. KDIGO Clinical Practice Guideline for Lipid Management in Chronic Kidney Disease. Kidney Int 2013; Suppl 3: 263.

4. Brennan DC, Lentine KL, Murphy B, Lam AQ. Lipid abnormalities after renal transplantation. UpToDate, Wolters Kluver 2017; 25.5.2$118-\mathrm{C} 25-251$.

5. Kassiske BL, Cangro CB, Hariharan $S$ et al. The evaluation of renal trans-plantation candidates: clinical practice guidelines. Am J Transplant 2001; Suppl 1: 2-3.

6. Brennan DC, Lentine KL, Murphy B, Lam AQ. Lipid abnormalities after renal transplantation. UpToDate, Wolters Kluver 2017; 25.5.2$118-\mathrm{C} 25-251$.

7. Hoefner DM, Hodel SD, O'Brien JF et al. Development of a rapid quantitative method for LDL subfractions with use of the Quantimetrix Lipoprint LDL system. Clin Chem 2001; 472: 266-274.

8. McNamara JR, Campos H, Ordovas JM et al. Effect of gender, age, and lipid status on low density lipoprotein subfraction distribution: results from the Framingham Offspring Study. Arteriosclerosis 1987; 7: 483-490.

9. Yeo FE, Willines TC, Bucci JR et al. Cardiovascular risk in stage 4 and 5 nefropathy. Adv Chronic Kidney Dis 2004; 11: 116.

10. Fruchart JCh, Sacks FM, Hermans MP et al. The residual risk reduction initiative: a call to action to reduce residual vascular risk in dyslipidemic patients. Diab Vasc Dis Res 2008; 5: 319-335.

11. Abramowicz D, Cochat P, Claas FH et al. European Best Clinical Practice Guideline on kidney donor and recipient evaluation and perioperative care. Nephrol Dial Transplant 2015; 30: 1790.

12. Ojo AO. Cardiovascular complications after renal transplantation and their prevention. Transplantation 2006; 82: 603.
13. Fried LF, Orchard TJ, Kasiske BL. Effect of lipid reduction on the progression of renal disease: a meta-analysis. Kidney Int 2001; 59: 260.

14. Holdaas H, Fellstrom B, Jardine AG et al. Beneficial effect of early initiation of lipid lowering therapy following renal transplantation. Nephrol Dial Transplant 2005: 20; 974.

15. Cosio FG, Hickson LJ, Griffin MD et al. Patient survival and cardiovascular risk after kidney transplantation: a challenge of diabetes. Am J Transplant 2008; 8: 593.

16. 2016 ESC/EAS Guidelines for the management of dyslipidaemias. Eur Heart J 2016. doi: 10.1093/eurheartj/ehw272.

17. Jardine AG, Holdaas H, Fellstrőm B et al. Fluvastatin prevents cardiac death and myocardial infarction in renal transplant recipients: post-hoc subgroup analyses of the ALERT Study. Am J Transplant 2004; 4: 988.

18. Navaneethan SD, Perkovic V, Johnson DW et al. HMG CoA reductase inhibitors (statins) for kidney transplant recipients. Cochrane Database Syst Rev 2009: CD 0050019.

19. Baigent C, Landray MJ, Reith $\mathbf{C}$ et al. The effects of lowering LDL cholesterol with simvastatin plus ezetimibe in patients with chronic kidney disease (Study of Heart and Renal Protection) a randomized placebocontrolled trial. Lancet 2011; 377: 2181.

20. Jellinger PS, Handelsman Y, Rosenblit PD et al. Guidelines for the management of dyslipidemia and prevention of cardiovascular disease. American Association of endocrinologists and American College of Endocrinology. AACE 2017 Guidelines. Endocrine Pract 2017; 23 (Suppl 2): $1-87$.

Received December 4, 2017. Accepted January 11, 2017. 